\title{
Enhancing Part Properties in Additive Manufacturing Through Material Design and Print Process Optimization
}

\author{
M. A. Aubart, S. Jain, D. Liu, A. Pedicini, and S. Serpe ${ }^{1}$ \\ 1. Arkema Inc., King of Prussia, PA, USA
}

Arkema Inc. is a global chemicals and materials manufacturing company that supplies materials for many key markets, with a strategic emphasis on providing solutions to address six emerging global trends, one of which is 3D printing. As such, Arkema is focused on strong R\&D initiatives in the next generation of printable materials, including a diverse array of amorphous and semi-crystalline thermoplastics for extrusion and powder fusion based processes, and radiation curable materials for jetting and stereolithography techniques. This talk will highlight select insights and results from Arkema R\&D on several of these additive manufacturing techniques, and Arkema material technologies including Kynar $^{\circledR}$ polyvinylidene fluoride, Plexiglas ${ }^{\circledR}$ acrylic, Rilsan $^{\circledR}$ and Orgasol ${ }^{\circledR}$ polyamides, Kepstan ${ }^{\circledR}$ polyetherketoneketone, and Sartomer radiation curable resin.

Stereolithography (SLA) is the oldest and most widely used industrial 3D printing technology.[1] It uses photo-curable liquid resins to build an object in a vat, layer by layer from a resin that cures on contact with laser light. SLA offers many advantages compared to other 3D Printing processes such as speed, surface finish, and the ability to build transparent objects. However, a key limitation can be the poor mechanical properties of the printed objects. Our background in UV curable specialty oligomers and monomers has allowed us to develop UV cure resins that exhibit physical properties after printing that are similar to thermoplastic materials. Typically, a photocurable resin formulation for stereolithography consists of an oligomer, reactive diluents, and a photo-initiator. Oligomers provide toughness and elongation while reactive monomers reduce viscosity and enhance print cure speed. This part of the talk will discuss select recent advances in oligomer design to improve the mechanical properties of printed objects and close the gap between thermoset and thermoplastic materials.

Due to relatively inexpensive and easy to operate machines, material extrusion printing is a familiar 3D printing technique to most consumers, most notably as fused deposition modeling (FDM).[2] The extrusion printing process typically relies on forcing a thermoplastic filament through an extrusion head as the build platform or the nozzle moves in the $x-y$ plane. Once a layer is deposited, the head or platform is lifted. The next layer is then deposited and joined to the previous layer, building up, layer by layer, a final printed part. Part challenges in this field generally revolve around obtaining optimal consolidation between layers to allow for high z-direction strength, and optimizing the print process. These factors are a focus in order to reach as closely as possible to the intrinsic bulk properties that can be achieved with the same material in a conventionally injection molded or extruded part. By utilizing our compositional toolbox and understanding of amorphous and semi-crystalline polymers, and polymer formulations, we've begun to map how material part characteristics can be optimized through the manipulation of rheology (e.g., melt viscosity), degree of crystallinity, and processing conditions. This part of the talk will discuss select recent advances in new developmental thermoplastics and process protocols specifically optimized for extrusion printing parts.

In addition to FDM and SLA materials, Arkema also conducts research and development on optimized polymeric materials for use in powder bed fusion, commonly referred to as selective laser sintering 
(SLS).[3] This technique relies on the laser induced fusion of thermoplastic semi-crystalline powders to build parts with excellent z-direction mechanical properties. In the process, the SLS machine is designed to distribute a surface layer of polymer powder over a part bed of additional powder held at a temperature just below where particle coalescence can occur. A laser then melts a pattern of the desired layer, "sintering" a slice of the part. A fresh layer of surface powder is then added and the process is repeated. Optimization of particle shape, size, and density along with print protocols are key to generating parts fabricated from high performance engineering materials (e.g., polyamides and polyetherketoneketones). This part of the talk will highlight select recent advances in recyclable powders for SLS processes.

\section{References:}

[1] FPW Melchels, J Feijen, and DW Grijpma, Biomaterials 31 (2010), p. 6121.

[2] OA Mohamed, SH Masood, and JL Bhowmik, Adv. Manuf. 3 (2015), p. 42.

[3] R Rajesh, S Sudheer, and MV Kulkarni, IJCESR 2 (2015), p. 2393.

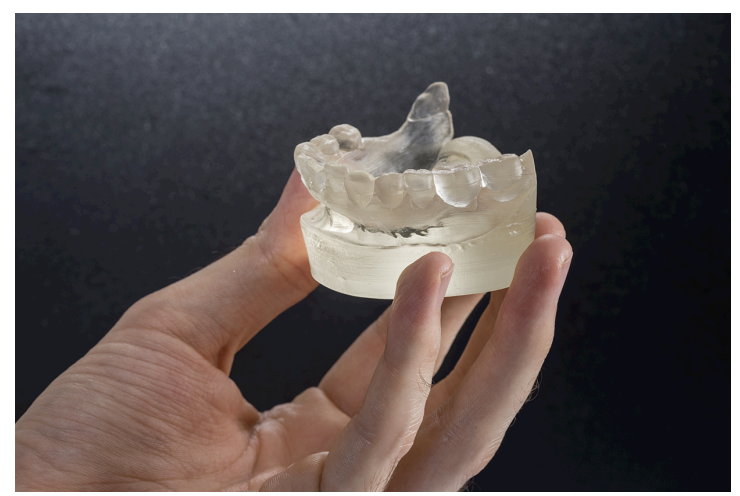

Figure 1. Typical SLA part printed at Arkema's AM laboratory using a proprietary resin formulation.

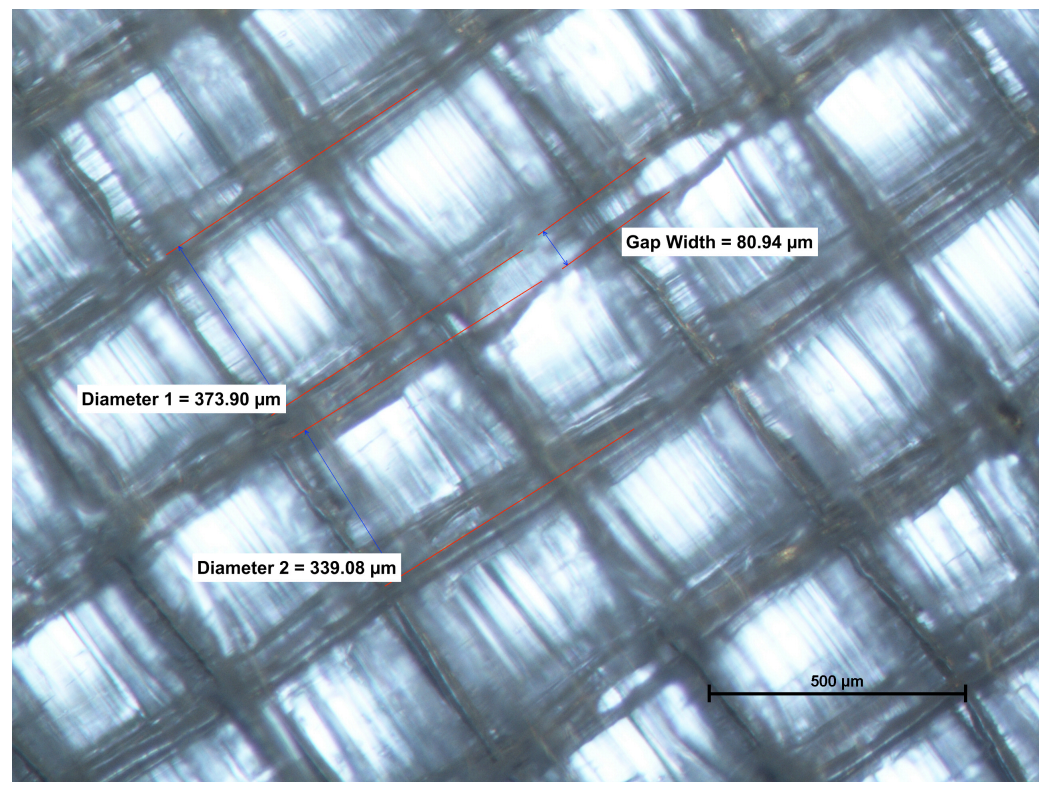

Figure 2. Optical micrograph of a flat part's top surface printed using standard extrusion printing conditions (400 micron nozzle) and a cross-wise molten filament process in Arkema's AM laboratory. Gap widths and diameter sizes result from shrinkage on freezing of the proprietary polymer. 